\title{
Helmintofauna Gastrointestinal de Importancia Zoonótica y sus Aspectos Patológicos en Roedores (Rattus spp) en Tres Medioambientes
}

\author{
Gastrointestinal Helminth Fauna of Zoonotic Importance and Pathological \\ ASPECTS IN RODENTs (Rattus Spp) In THREE ENVIRONMENTS
}

\author{
Deisy Abad A. ${ }^{1}$, Amanda Chávez V. ${ }^{1,4}$, Rosa Pinedo V. ${ }^{1}$, Manuel Tantaleán V. ${ }^{2}$, \\ Omar Gonzáles-Viera ${ }^{3}$
}

\section{RESUMEN}

\begin{abstract}
El objetivo del estudio fue identificar y determinar la prevalencia de helmintos gastrointestinales de importancia zoonótica presentes en roedores (Rattus spp) en tres medioambientes (granjas porcinas, zoológico y mercados de abastos) de Lima, Perú, así como estimar la asociación entre presencia de helmintos y las variables lugar de procedencia, especie, edad y sexo. Se capturaron 245 (Rattus rattus y Rattus norvergicus) roedores mediante el uso de trampas de captura viva. Cráneos, piel, carta dentaria y parámetros morfométricos se utilizaron para la clasificación taxonómica de las especies. Se colectó el estómago y el intestino delgado y grueso. Los helmintos se colocaron en alcohol $70 \%$ y los tejidos fueron preservados en formol 10\%. La prevalencia de helmintos gastrointestinales fue de $72.2 \%$, en tanto que la prevalencia de helmintos zoonóticos fue de $46.5 \%$. Se hallaron cuatro especies de importancia zoonótica: Raillietina demerariensis, Gongylonema neoplasticum, Hymenolepis diminuta y Moniliformis moniliformis; estos dos últimos de mayor frecuencia en los tres ecosistemas. Otros helmintos identificados fueron Mastophorus muris, Heterakis spumosa, Aspicularis tetrapera, Syphacia muris, Trichuris muris, Capillaria sp y Vampirolepis fraterna. Los hallazgos histopatológicos mostraron disminución del tamaño de las vellosidades intestinales, desprendimiento de células epiteliales, aumento del número de células caliciformes y enterocitos con presencia de eosinófilos, linfocitos, plasmocitos y macrófagos asociados a H. diminuta, M. moniliformis y $R$. demerariensis.
\end{abstract}

Palabras clave: Rattus rattus, Rattus norvegicus, zoonosis, zoológico, granjas porcinas, mercados

\footnotetext{
${ }^{1}$ Laboratorio de Parasitología y Microbiología, Facultad de Medicina Veterinaria, Universidad Nacional Mayor de San Marcos, Lima, Perú

${ }^{2}$ Laboratorio de Parasitología de Fauna Silvestre y Zoonosis, Facultad de Biología, Universidad Nacional Mayor de San Marcos, Lima, Perú

${ }^{3}$ Facultad de Medicina Veterinaria, Universidad de California, Davis, Estados Unidos

${ }^{4}$ E-mail: achavezvg@gmail.com
}

Recibido: 30 de marzo de 2016

Aceptado para publicación: 31 de agosto de 2016 
The study aimed to identify and determine the prevalence of zoonotic gastrointestinal helminths present in rodents (Rattus spp) in three environments (pig farms, zoo and food markets) of Lima, Peru and estimate the association between the presence of helminths and the variables place of origin, species, age and sex. A total of 245 rodents (Rattus rattus and Rattus norvergicus) were captured alive using live trap cages and were subsequently euthanized. Skulls, skin, teeth and morphometric parameters were used for taxonomic classification of species. Stomach and small and large intestine were collected. The helminths found were placed in 70\% alcohol and selected tissues were preserved in $10 \%$ formalin. The prevalence of gastrointestinal helminths in rats was $72.2 \%$ whereas the prevalence of zoonotic helminth was $46.5 \%$. Four species of zoonotic importance were found: Raillietina demerariensis, Gongylonema neoplasticum, Hymenolepis diminuta and Moniliformis moniliformis, where the latter two were more frequent in the three ecosystems. Other helminths identified were Mastophorus muris, Heterakis spumosa, Aspicularis tetrapera, Syphacia muris, Trichuris muris, Capillaria sp and Vampirolepis fraterna. The histopathological findings included a decrease in size of the villi, detachment of epithelial cells, increased numbers of goblet cells and enterocytes with eosinophils, lymphocytes, plasma cells and macrophages associated with $H$. diminuta, M. moniliformis and $R$. demerariensis.

Key words: Rattus rattus, Rattus norvegicus, zoonoses, zoo, pig farms, markets

\section{INTRODUCCIÓN}

Los roedores (Rattus spp) constituyen especies dominantes, encontrándose en la mayoría de las regiones del mundo debido a su gran capacidad de adaptación a diversos ecosistemas (Paramasvaran et al., 2009). Así mismo, revisten de importancia en la cadena epidemiológica pues son piezas para la transmisión de diversas enfermedades parasitarias en animales silvestres y domésticos, incluido el hombre (Rodríguez et al., 2010).

Parásitos como Hymenolepis diminuta, Moniliformis moniliformis, Gongylonema neoplasticum, Raiilietina demeranensis y Trichinella spiralis se encuentran en los roedores, donde estos actuarían como reservorios para la transmisión al hombre (Parshad, 1999; Stojcevic et al., 2004). Es así que el incremento de la población de roedores en un área puede estar directamente relacionado con el aumento de las enfermedades zoonóticas en la población humana. $\mathrm{La}$ infección en el hombre es principalmente a través de la ingestión de alimentos y agua contaminados con heces de roedores e indirectamente a través de la ingestión accidental de vectores (Bowman, 2011; Pakdel et $a l ., 2013)$.

En el Perú son escasos los estudios sobre fauna parasitaria de roedores y su rol como posible transmisor o reservorio de parásitos gastrointestinales con impacto en la salud pública. Esta escasez de información científica demanda investigar la actual distribución y frecuencia de estos parásitos en roedores bajo diferentes ecosistemas, a fin de determinar el riesgo potencial que corren las poblaciones de animales y el hombre. 
Los objetivos del estudio fueron identificar y determinar la prevalencia de helmintos gastrointestinales de importancia zoonótica presentes en roedores (Rattus spp) de tres medioambientes (granjas porcinas, zoológico y mercados de abastos) de Lima, estimar la asociación entre la presencia de helmintos y las variables lugar de procedencia, especie, edad y sexo, y describir las lesiones histopatológicas generadas en los hospederos.

\section{Materiales y Métodos}

El estudio se realizó en tres medioambientes: tres granjas porcinas ubicadas en los distritos de Villa El Salvador, Lurín y Huaral, el zoológico del Patronato del Parque de las Leyendas, ubicado en el distrito de San Miguel, y en nueve mercados de abastos de los distritos de San Martín de Porres, La Victoria, San Juan de Miraflores, Chorrillos y Surco, durante el periodo comprendido entre febrero de 2013 y julio de 2015.

El tamaño de la muestra (91 ratas por ecosistema) se calculó por medio de la fórmula de poblaciones infinitas (Daniel, 1996), con un nivel de confianza del $95 \%$, un error del $5 \%$ y una prevalencia referencial de 93.75\% (Iannacone y Alvariño, 2002).

Se utilizaron trampas «Tomahawk» de captura viva para ratas con ayuda de cebos no tóxicos (choclo, pepa de zapallo, carne molida, esencia de vainilla, fruta y tomate). Se registró el sexo, la procedencia y los parámetros morfométricos (longitud total, largo de cola, patas y orejas y el peso) para establecer la especie. Como edad se consideraron dos categorías: juvenil-subadulto y adulto, donde los roedores Rattus rattus se clasificaron como adultos con un peso mayor de $130 \mathrm{~g}$ (Milazzo et al., 2010) y los Rattus norvegicus con un peso mayor de 200 g (Kataranovski et al., 1994).
Los animales fueron trasladados vivos hacia áreas aisladas, especialmente dispuestas por el zoológico y por la Facultad de Medicina Veterinaria (FMV) de la Universidad Nacional Mayor de San Marcos (UNMSM), para su manipulación según estándares de bioseguridad y normas de procesamiento acordes a los protocolos del Centro de Enfermedades Infecciosas y Prevención de Atlanta (Mills et al., 1998). Los roedores fueron anestesiados mediante inhalación de cloroformo e inyección de ketamina $(7.5 \mathrm{mg} /$ $\mathrm{kg}$ ) y sacrificados con sobredosis de pentobarbital sódico (Mills et al., 1998).

En la necropsia de las ratas se recolectó el tracto gastrointestinal. Los extremos de cada tracto fueron ligados y fijados en alcohol-formol-ácido acético (AFA), preparado según las recomendaciones de Botero y Restrepo (2006). Se realizó la observación y colección in situ de los helmintos de mayor tamaño y, luego, el contenido gastrointestinal fue pasado por un tamiz de $80 \mu \mathrm{m}$, permitiendo la colecta directa de helmintos pequeños con la ayuda del estereoscopio. Los helmintos fueron fijados con alcohol al 70\% y llevados al Laboratorio de Parasitología de Fauna Silvestre y Zoonosis de la Facultad de Biología de la UNMSM para su identificación morfológica. Las especies de nematodos se identificaron según las claves y descripciones descritas por Anderson et al. (2009) y los cestodos por Khalil et al. (1994). Asimismo, se seccionaron áreas de $1 \mathrm{~cm}^{2}$ del tracto gastrointestinal que presentaron lesiones y se preservaron en formol al 10\% para su estudio histopatológico.

Nematodos y acantocéfalos se revisaron con aclarante alcohol-fenol. Los cestodos fueron aplanados entre dos láminas portaobjetos y colocados en un frasco de boca ancha con alcohol al 70\% para luego ser deshidratados mediante una batería de alcoholes, coloreados con carmín acético de Semichon y montados en bálsamo de Canadá. 
Cuadro 1. Prevalencia (porcentaje \pm intervalo de confianza) y asociación entre el parasitismo gastrointestinal y las variables medioambiente, especie, sexo y edad en ratas (Rattus spp) de Lima, Perú (2015)

\begin{tabular}{|c|c|c|c|c|c|c|}
\hline & \multirow{2}{*}{ N. ${ }^{\circ}$} & \multirow{2}{*}{$\begin{array}{c}\text { Positivos } \\
\% \pm \mathrm{IC}\end{array}$} & \multirow{2}{*}{$X^{2}$} & \multirow{2}{*}{$\begin{array}{l}\text { Valor } \\
\text { de p }\end{array}$} & \multicolumn{2}{|c|}{ Odds Ratio } \\
\hline & & & & & Valor & IC $95 \%$ \\
\hline \multicolumn{7}{|l|}{ Medioambiente } \\
\hline Granja porcina & 91 & $93.4 \pm 5.1$ & - & - & - & - \\
\hline Zoológico & 91 & $78.0 \pm 8.5$ & - & - & 2.82 & $1.0-7.9$ \\
\hline Mercado de abastos & 63 & $33.3 \pm 11.6$ & 69.41 & 0.000 & 27.29 & $9.6-77.7$ \\
\hline \multicolumn{7}{|l|}{ Especie } \\
\hline Rattus rattus & 150 & $65.3 \pm 7.6$ & - & - & - & - \\
\hline Rattus norvegicus & 95 & $83.2 \pm 7.5$ & 9.22 & 0.002 & 0.95 & $0.4-2.1$ \\
\hline \multicolumn{7}{|l|}{ Sexo } \\
\hline Hembra & 129 & $72.9 \pm 7.6$ & - & - & - & - \\
\hline Macho & 116 & $71.6 \pm 8.2$ & 0.05 & 0.82 & 0.78 & $0.4-1.5$ \\
\hline \multicolumn{7}{|l|}{ Edad } \\
\hline Juvenil-subadulto & 127 & $66.9 \pm 8.2$ & - & - & - & - \\
\hline Adulto & 118 & $78.0 \pm 7.5$ & 3.72 & 0.054 & 0.44 & $0.2-1.0$ \\
\hline Total & 245 & $72.2 \pm 5.6$ & & & & \\
\hline
\end{tabular}

Los tejidos seleccionados fueron procesados histológicamente y teñidos con hematoxilina y eosina (HE), siguiendo las técnicas recomendadas por Behmer et al. (1976), a fin de observar posibles lesiones histopatológicas.

Se calculó la prevalencia de helmintos y se expresó en forma porcentual con sus respectivos intervalos de confianza al 95\% (Daniel, 1996). La posible asociación y riesgo de infección $(\mathrm{p}<0.05)$ entre las variables especie, sexo, edad y procedencia de las ratas con el parasitismo se analizó mediante la prueba de Chi cuadrado y Odds Ratio, respectivamente, utilizando el paquete estadístico Stata v. 12.0.

Todos los procedimientos del presente estudio contemplaron los lineamientos de las buenas prácticas clínicas y de ética en in- vestigación biomédica, en cumplimiento con las normas reglamentadas por el Comité de Ética para el Uso de Animales de Experimentación de la FMV-UNMSM (Expediente 2014-002).

\section{Resultados y Discusión}

La prevalencia de helmintos gastrotestinales en ratas de Lima procedentes de tres medioambientes fue de $72.2 \pm 5.6 \%$ (intervalo de confianza del 95\%) (Cuadro 1). La mayor prevalencia de helmintos se observó en los centros de producción porcina (93.4 $\pm 5.1 \%$ ). En mercados solo se pudo capturar 63 roedores ( $58 R$. rattus y $5 R$. norvegicus) de las 91 requeridos, a pesar de que se hicieron las capturas en nueve mercados, de allí que se determinó el $6 \%$ de error máximo 
admisible para este tamaño muestral (Daniel, 1996). La prueba de Chi cuadrado determinó asociación significativa entre la variable procedencia y la presentación del parasitismo $(\mathrm{p}=0.000)($ Cuadro 1$)$.

La prevalencia de helmintos gastrointestinales para las variables especie, sexo y edad se muestran en el Cuadro 1, habiéndose encontrado asociación significativa únicamente con la variable especie $(\mathrm{p}=0.002)$.

Se registraron siete nematodos $(55.5 \pm$ $6.2 \%)$, tres especies de cestodos $(35.1 \pm 6 \%)$ $\mathrm{y}$ un acantocephalo $(22.0 \pm 5.2 \%)$. Hymenolepis diminuta $(25.7 \pm 5.5 \%)$ fue el helminto con la frecuencia más alta (Cuadro 2, Figura 1). Asimismo, se identificaron cuatro tipos de asociaciones parasitarias, donde el $43.5 \%$ de animales estuvo parasitado con un solo helminto (monoparasitismo), $35.6 \%$ con biparasitismo, $13.6 \%$ con triparasitismo y $4 \%$ con tetraparasitismo.

La prevalencia de helmintos de importancia zoonótica encontrada fue de $46.5 \pm$ $6.2 \%(114 / 245)$, habiendo asociaciones parasitarias en algunos roedores. Se reporta cuatro especies: Hymenolepis diminuta $(25.7 \pm 5.5 \%)$, Moniliformis moniliformis $(22.0 \pm 5.2 \%$; Figura 2C), Gongylonema neoplasticum $(6.1 \pm 3 \%$; Figura 3$) \mathrm{y}$ Raillietina demerariensis ( $4.1 \pm 2.5 \%$; Figura 2A) (Cuadro 2).

En las granjas porcinas se determinó la presencia de 10 especies de helmintos, en el zoológico fue de siete especies y en los mercados de abastos igualmente de siete especies (Cuadro 2). El único espécimen de Capillaria sp fue una hembra grávida a nivel del intestino delgado, sin poder llegar a identificar la especie.

Las lesiones histopatológicas en los tejidos afectados por los helmintos se muestran en el Cuadro 3.
El alto número de helmintos identificados en el presente estudio se debe, probablemente, al muestreo de varios medioambientes en diferentes áreas de la ciudad de Lima, mostrando una visión más general y próxima a la realidad sobre la diversidad parasitaria en estas ratas. Estudios previos en distritos de Lima en roedores capturados en viviendas, mercados de abastos y zonas aledañas al río Rímac conducidos por Ayulo y Dammert (1947), Iannacone y Alvariño (2002), Romero et al. (2012) y De Sotomayor et al. (2015) encontraron prevalencias de 76.4, 93.8, 83.6 y $83.6 \%$ y una riqueza parasitaria de $7,4,9$ y 10 helmintos, respectivamente. Así mismo, prevalencias similares al presente estudio fueron obtenidas por Stojcevic et al. (2004) en dos áreas rurales de Croacia $(72.6 \%$ y 8 helmintos), Kataranovski et al. (2011) en áreas urbanas y rural-suburbana de Belgrado, Serbia (68.5\% y 7 helmintos) y Mohd et al. (2012) en Kuala Lumpur, Malasia (80\% y 11 helmintos).

Hymenolepis diminuta (Figura 1) fue el helminto de mayor prevalencia general $(25.7 \%)$ y en granjas porcinas $(53.8 \%)$; sin embargo, en el zoológico y los mercados de abastos se encontraron frecuencias muy bajas ( 8.8 y $9.5 \%$, respectivamente), posiblemente debido a la mayor presencia de hospedadores intermediarios (escarabajos, pulgas, orugas y otros insectos) donde se almacenan granos, semillas y alimentos secos por tiempo prolongado y, que al mismo tiempo, son los alimentos favoritos para las ratas. Resultados similares fueron hallados por Tung et al. (2008) en granjas (38.9\%) y mercados (9.3\%) de Taiwan (Tung et al., 2013).

Los primeros reportes en Lima sobre Moniliformis moniliformis (Figura 2C) fueron realizados por Ayulo y Dammert (1947) en un estudio de 1000 R. norvegicus $(15.5 \%)$. Por otro lado, Tantaleán et al. (2005) solo reporta su presencia en $R$. rattus, $R$. rattus alexandrinus y Saimiri sciureus en Lambayeque, Lima e Iquitos, mientras que 
Cuadro 2. Prevalencia (porcentaje \pm intervalo de confianza) de helmintos gastrointestinales en ratas, según el medioambiente, en Lima-Perú (2015)

\begin{tabular}{|c|c|c|c|c|}
\hline & \multicolumn{3}{|c|}{ Medioambientes } & \multirow[b]{2}{*}{ Total } \\
\hline & $\begin{array}{l}\text { Granjas } \\
\text { porcinas }\end{array}$ & Zoológico & $\begin{array}{l}\text { Mercados } \\
\text { de abastos }\end{array}$ & \\
\hline \multicolumn{5}{|l|}{ Nematodos } \\
\hline Mastophorus muris & 7.7 & 4.4 & 0 & 4.5 \\
\hline Gongylonema neoplasticum* & 4.4 & 9.9 & 3.2 & 6.1 \\
\hline Capillaria sp. (intestinal) & 1.1 & 0 & 0 & 0.4 \\
\hline Heterakis spumosa & 31.9 & 27.5 & 0 & 22.0 \\
\hline Aspiculuris tetrapera & 38.5 & 0 & 1.6 & 14.7 \\
\hline Syphacia muris & 20.9 & 37.4 & 1.6 & 22.0 \\
\hline Trichuris muris & 0 & 0 & 3.2 & 0.8 \\
\hline Sub-total $(\% \pm$ IC $95 \%)$ & $74.5 \pm 9.0$ & $68.1 \pm 9.6$ & $9.5 \pm 7.2$ & $55.5 \pm 6.2$ \\
\hline \multicolumn{5}{|l|}{ Cestodos } \\
\hline Hymenolepis diminuta* & 53.8 & 8.8 & 9.5 & 25.7 \\
\hline Vampirolepis fraterna & 12.1 & 0 & 6.3 & 6.1 \\
\hline Raillietina demerariensis* & 9.9 & 1.1 & 0 & 4.1 \\
\hline Sub-total $(\% \pm$ IC 95\%) & $73.6 \pm 9.1$ & $9.9 \pm 6.1$ & $15.9 \pm 9.0$ & $35.1 \pm 6.0$ \\
\hline \multicolumn{5}{|l|}{ Acanthocephalo } \\
\hline Moniliformis moniliformis* & $9.9 \pm 6.1$ & $38.5 \pm 10.0$ & $15.9 \pm 9.0$ & $22.0 \pm 5.2$ \\
\hline Total $(\% \pm$ IC $95 \%)$ & $93.4 \pm 5.1$ & $78.0 \pm 8.5$ & $33.3 \pm 11.6$ & $72.2 \pm 5.6$ \\
\hline
\end{tabular}

* Helmintos de potencial zoonótico

Ibañez et al. (2005) lo reportó en $R$. norvegicus en el departamento de Amazonas. Recientemente, De Sotomayor et al. (2015) reportó el $32.9 \%$ de prevalencia en 53 ratas de cinco distritos de Lima.

Resultados variados sobre la presencia de Gongylonema neoplasticum (Figura 3) han sido reportados en mercados de Lima. Así, Romero et al. (2012) halló 3.3\% de prevalencia en ratas del distrito del Rímac en tanto que De Sotomayor et al. (2015) reportó $50.7 \%$ en ratas capturadas en cinco distritos. La infección en un hombre fue reportada por primera vez por Haruki et al. (2005) en Japón, donde la infección habría ocurrido por la ingestión accidental del hospedero in- termediario (cucaracha o escarabajo) contenida en verduras o agua.

Raillietina demerariensis (Figura 2A) solo fue hallada en las granjas porcinas $(9.9 \%)$ y en el zoológico (1.1\%). Este cestodo ha sido reportado en el país en capibaras (Hydrochaeris hydrochaeris) del Manú (Tantaleán, 1994) y en $R$. norvegicus en la ribera del río Rímac (Martínez y Tantaleán, 1998). Más recientemente, De Sotomayor et al. (2015) reportaron una frecuencia de 5.5\% en ratas de cinco distritos limeños.

La presencia de Vampirolepis fraterna (Figura 2B) en granjas porcinas $(12.1 \%)$ y mercados de abastos (6.3\%) en bajas fre- 
Cuadro 3. Lesiones histopatológicas causadas por helmintos en tractos gastrointestinales de ratas (Rattus rattus, Rattus norvegicus) de Lima, Perú (2015)

\begin{tabular}{|c|c|c|c|c|c|}
\hline \multirow[b]{2}{*}{ Helminto } & \multirow[b]{2}{*}{$\begin{array}{l}\text { Órgano } \\
\text { (n) }\end{array}$} & \multirow[b]{2}{*}{ Diagnóstico histopatológico } & \multicolumn{3}{|c|}{$\begin{array}{l}\text { Grado de } \\
\text { infección }\end{array}$} \\
\hline & & & בัּ & $\begin{array}{l}\frac{0}{\pi} \\
\frac{\pi}{0} \\
\frac{0}{2} \\
\sum\end{array}$ & 苛 \\
\hline \multirow[t]{2}{*}{$\begin{array}{l}\text { Hymenolepis } \\
\text { diminuta }\end{array}$} & $\begin{array}{l}\text { Duodeno } \\
\text { (2) }\end{array}$ & $\begin{array}{l}\text { Duodenitis catarral difusa aguda con } \\
\text { atrofia de vellosidades intestinales e } \\
\text { hiperplasia de enterocitos y células } \\
\text { caliciformes }\end{array}$ & 1 & 1 & - \\
\hline & $\begin{array}{l}\text { Yeyuno } \\
\text { (3) }\end{array}$ & $\begin{array}{l}\text { Yeyunitis catarral difusa aguda con atrofia } \\
\text { de vellosidades intestinales e hiperplasia } \\
\text { de enterocitos y células caliciformes }\end{array}$ & 1 & 2 & - \\
\hline \multirow[t]{2}{*}{$\begin{array}{l}\text { Moniliformis } \\
\text { moniliformis }\end{array}$} & $\begin{array}{l}\text { Yeyuno } \\
(11)\end{array}$ & $\begin{array}{l}\text { Yeyunitis macrocítica difusa crónica con } \\
\text { atrofia de las vellosidades intestinales e } \\
\text { hiperplasia de enterocitos y células } \\
\text { caliciformes }\end{array}$ & - & 7 & 4 \\
\hline & $\begin{array}{l}\text { Yeyuno } \\
\text { (2) }\end{array}$ & $\begin{array}{l}\text { Yeyunitis linfoplasmocítica difusa aguda } \\
\text { con atrofia de las vellosidades intestinales } \\
\text { e hiperplasia de enterocitos y células } \\
\text { caliciformes }\end{array}$ & 2 & - & - \\
\hline $\begin{array}{l}\text { Raillietina } \\
\text { demerariensis }\end{array}$ & $\begin{array}{l}\text { Yeyuno } \\
(2)\end{array}$ & $\begin{array}{l}\text { Yeyunitis catarral eosinofílica difusa } \\
\text { aguda con hiperplasia de células } \\
\text { caliciformes. }\end{array}$ & - & 2 & - \\
\hline $\begin{array}{l}\text { Gongylonema } \\
\text { neoplasticum }\end{array}$ & $\begin{array}{l}\text { Estómago } \\
(8)\end{array}$ & $\begin{array}{l}\text { Gastritis eosinofílica difusa aguda con } \\
\text { hiperqueratosis. }\end{array}$ & 2 & 3 & 3 \\
\hline $\begin{array}{l}\text { Mastophorus } \\
\text { muris }\end{array}$ & $\begin{array}{l}\text { Estómago } \\
\text { (2) }\end{array}$ & Sin lesiones & - & - & - \\
\hline $\begin{array}{l}\text { Vampirolepis } \\
\text { fraterna }\end{array}$ & $\begin{array}{l}\text { Yeyuno } \\
\text { (3) }\end{array}$ & $\begin{array}{l}\text { Yeyunitis catarral linfoplasmocítica } \\
\text { multifocal crónica con hiperplasia de las } \\
\text { células caliciformes }\end{array}$ & 3 & - & - \\
\hline $\begin{array}{l}\text { Heterakis } \\
\text { spumosa }\end{array}$ & $\begin{array}{l}\text { Colon } \\
(6)\end{array}$ & $\begin{array}{l}\text { Colitis eosinofilica linfoplasmocítica } \\
\text { difusa aguda con hiperplasia folicular de } \\
\text { los agregados linfoides }\end{array}$ & 2 & 4 & - \\
\hline $\begin{array}{l}\text { Trichuris } \\
\text { muris }\end{array}$ & $\begin{array}{l}\text { Ciego } \\
(2)\end{array}$ & Tiflitis linfoplasmocítica difusa aguda & 2 & - & - \\
\hline $\begin{array}{l}\text { Aspiculuris } \\
\text { tetraptera }\end{array}$ & $\begin{array}{l}\text { Colon } \\
\text { (2) }\end{array}$ & $\begin{array}{l}\text { Colitis catarral linfocítica difusa crónica } \\
\text { con hiperplasia de agregados linfoides }\end{array}$ & - & 1 & 1 \\
\hline $\begin{array}{l}\text { Syphacia } \\
\text { muris }\end{array}$ & $\begin{array}{l}\text { Ciego } \\
\text { Colon } \\
\text { (3) }\end{array}$ & Sin lesiones & - & - & - \\
\hline
\end{tabular}



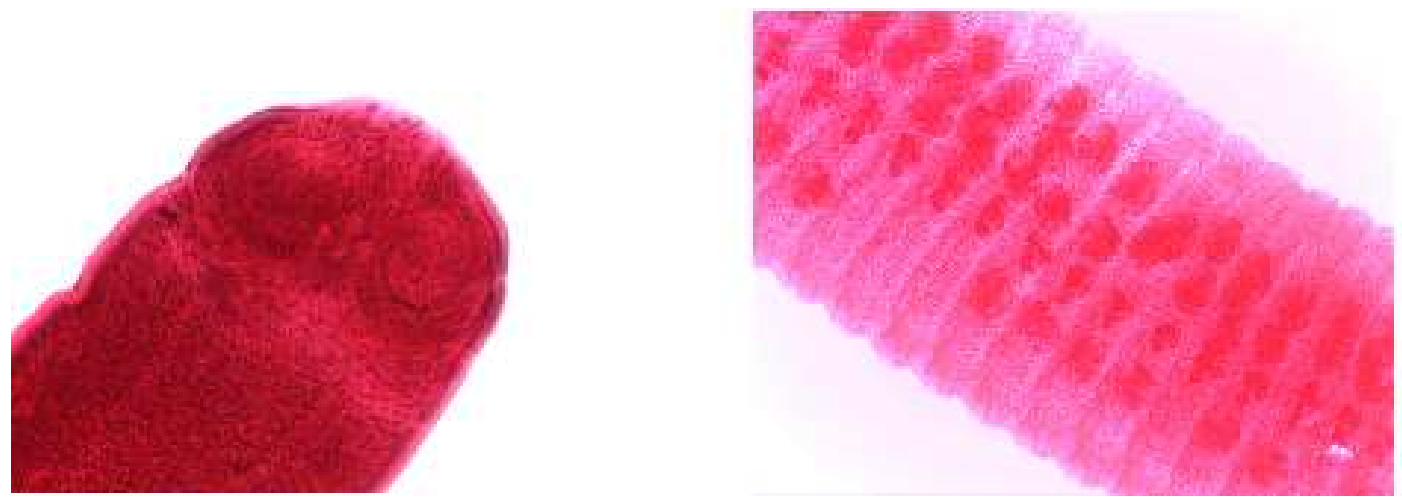

Figura 1. Hymenolepis diminuta. A) Vista lateral de la región anterior. Nótese la presencia de ventosas y escólex sin ganchos (400X). B) Proglótido maduro (400X). Tinción con carmín acético de Semichon

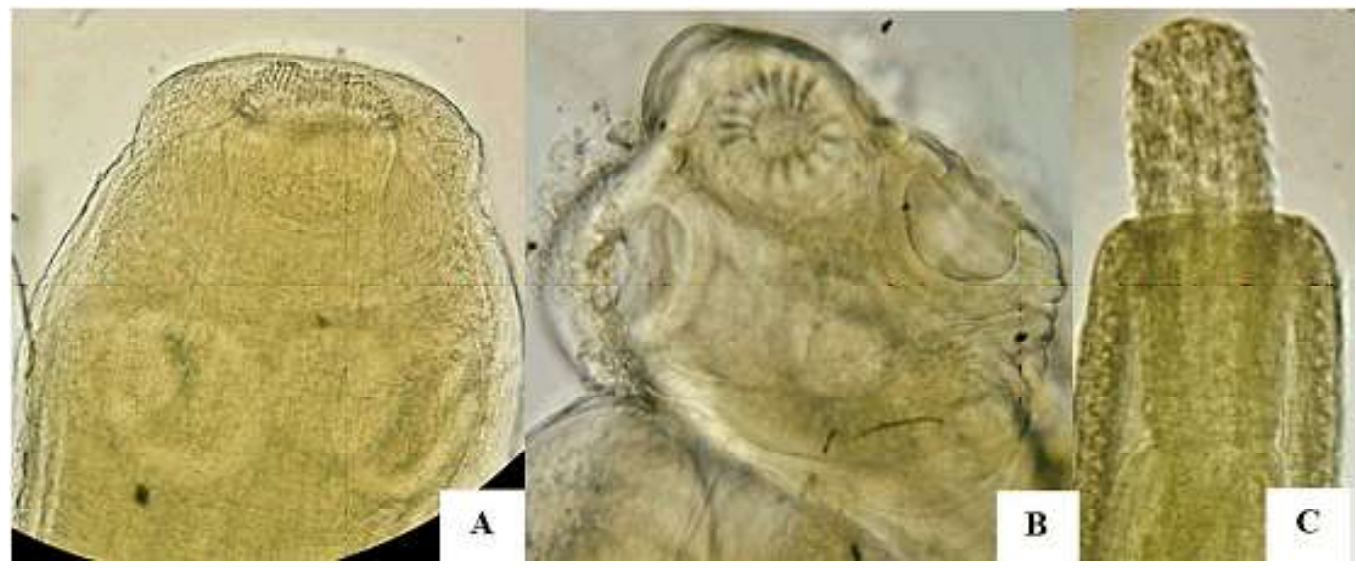

Figura 2. A) Raillietina demerariensis. Escólex con rostelo armado. Nótese la presencia de ganchos pequeños y cuatro ventosas (400X). B) Vampirolepis fraterna. Escólex con rostelo armado, observándose ganchos prominentes y las cuatro ventosas desarmadas (400X). C) Moniliformis moniliformis, vista lateral del extremo anterior del parásito. Nótese la presencia de ganchos en la probóscide (100X)

cuencias es coincidente con resultados previos en mercados de Lima con frecuencias de 6 a 11.5\% (Ayulo y Dammert, 1947; Romero et al., 2012; De Sotomayor et al., 2015). Asimismo, Tung et al. (2008) reportan solo $1.2 \%$ en Rattus norvegicus de granjas mientras que se obtuvieron $21.9 \%$ en $R$. norvegicus, $9.1 \%$ en $R$. rattus y $87.5 \%$ en el ratón Suncus murinus en mercados de
Taiwán (Tung et al., 2013). Históricamente, se consideró que primates y seres humanos eran susceptibles a las cepas de $V$. fraterna de roedores; sin embargo, estudios recientes han demostrado que las cepas que infectan roedores y las que infectan humanos son diferentes y no hay infección cruzada (Macnish, 2002; El-Sayad y Lotfy, 2005; Baker, 2007). 

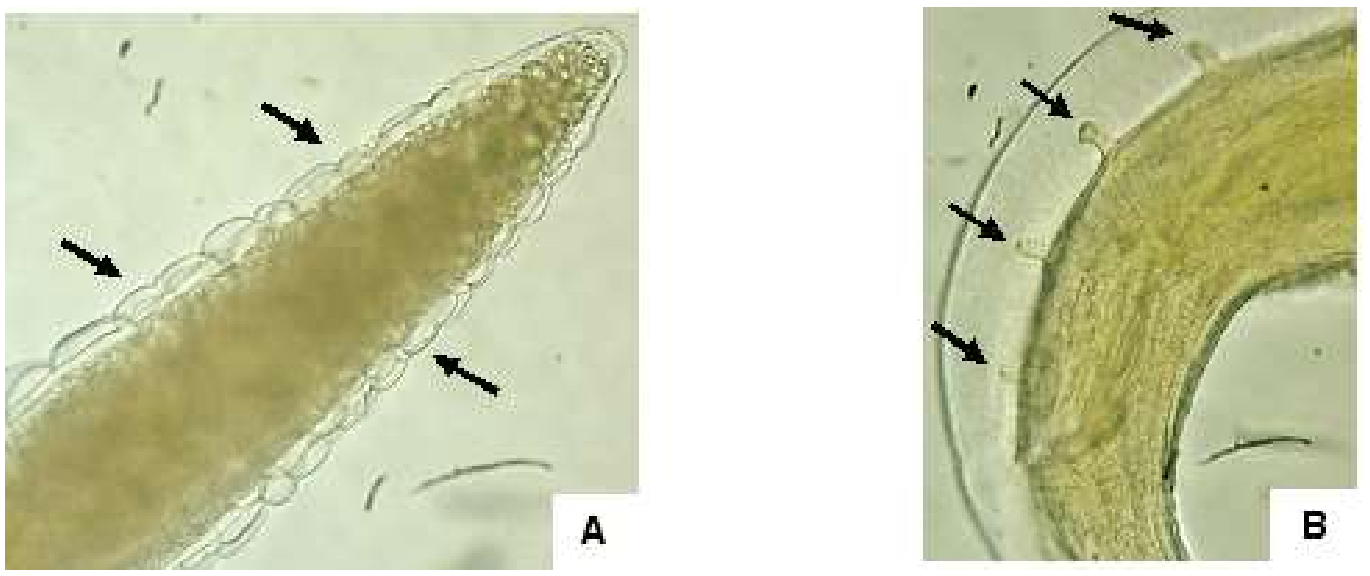

Figura 3. Gongylonema neoplasticum. A) Región anterior de Gongylonema neoplasticum. Vista lateral. Nótese las grandes protuberancias globulares (flechas) (100X). B) Región posterior del macho de Gongylonema neoplasticum. Vista lateral. Nótese las papilas caudales preanales (flechas) $(100 \mathrm{X})$

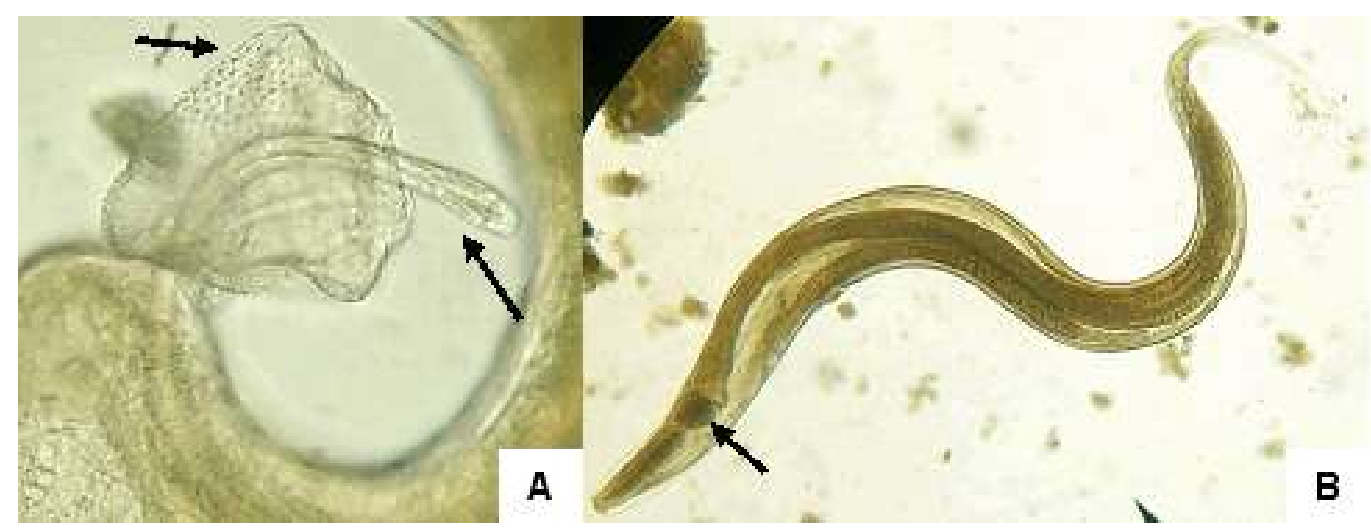

Figura 4. A) Trichuris muris. Vista lateral de la región posterior del macho. Nótese la espícula rodeada por una vaina con espinas cuticulares (flechas) (100X). B) Syphacia muris. Vista lateral de una hembra grávida. Nótese la forma del bulbo esofágico (flecha) (40X)

Los nematodos S. muris, H. spumosa y A. tetraptera (Figuras 4B, 5 y 6 , respectivamente) son parásitos típicos del género Rattus. El ciclo de vida monoxeno, natural de estos nematodos, posiblemente favorezca que este grupo de parásitos sea el dominante dentro de la comunidad de helmintos de pequeños mamíferos, especialmente roedores. Parásitos con un ciclo de vida simple y direc- to tienen más oportunidades de continuar la dispersión por sus hospedadores en comparación con los parásitos de ciclo de vida indirecto (Gómez-Villafañe et al., 2008; Kataranovski et al., 2011). Por otro lado, la baja frecuencia de Trichuris muris (Figura 4A) sería atribuido a la existencia de una fuerte respuesta inmune por parte del hospedador contra esta especie (Kataranovski et al., 2011). 

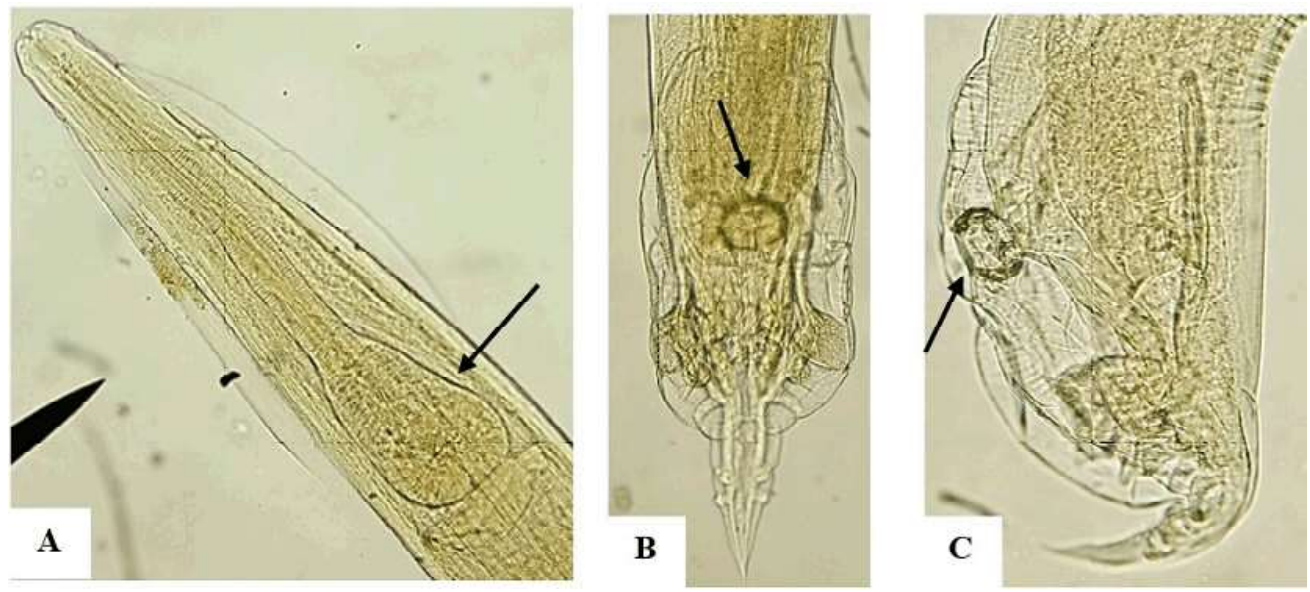

Figura 5. Heterakis spumosa. A) Vista lateral de la región anterior. Nótese la forma del bulbo esofágico (flecha) (100X). B, C) Vista ventral y lateral de la región posterior del macho, respectivamente. Obsérvese la ventosa genital (flechas) (100X)

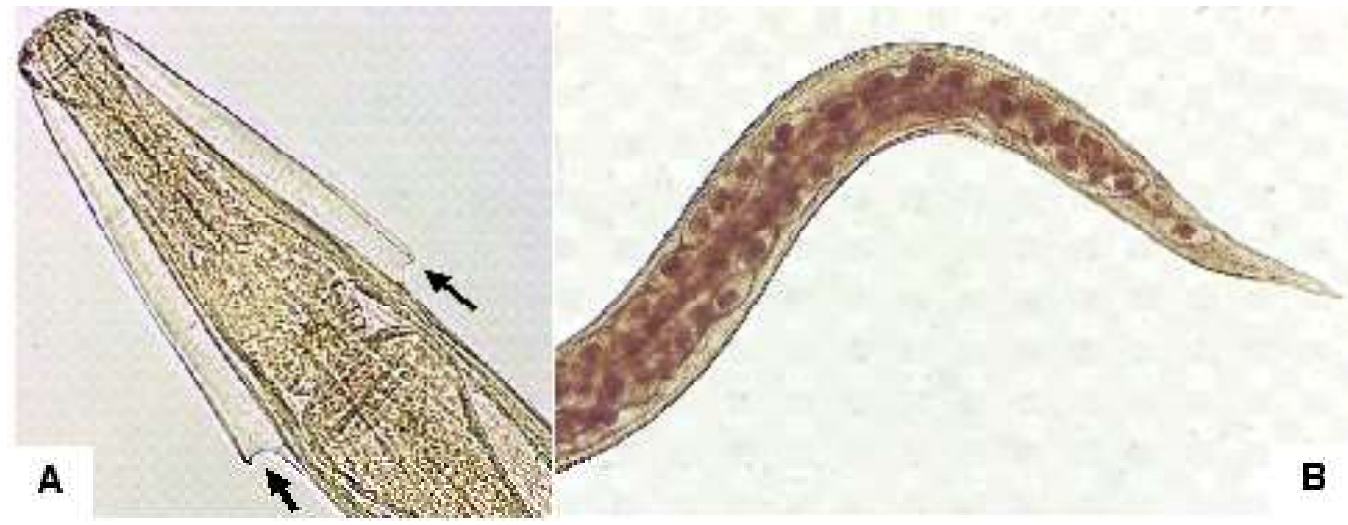

Figura 6. Aspiculuris tetraptera. A) Vista lateral de la región anterior. Nótese la terminación de las aleas cervicales (flechas) (100X). B) Vista lateral de una hembra grávida $(100 \mathrm{X})$
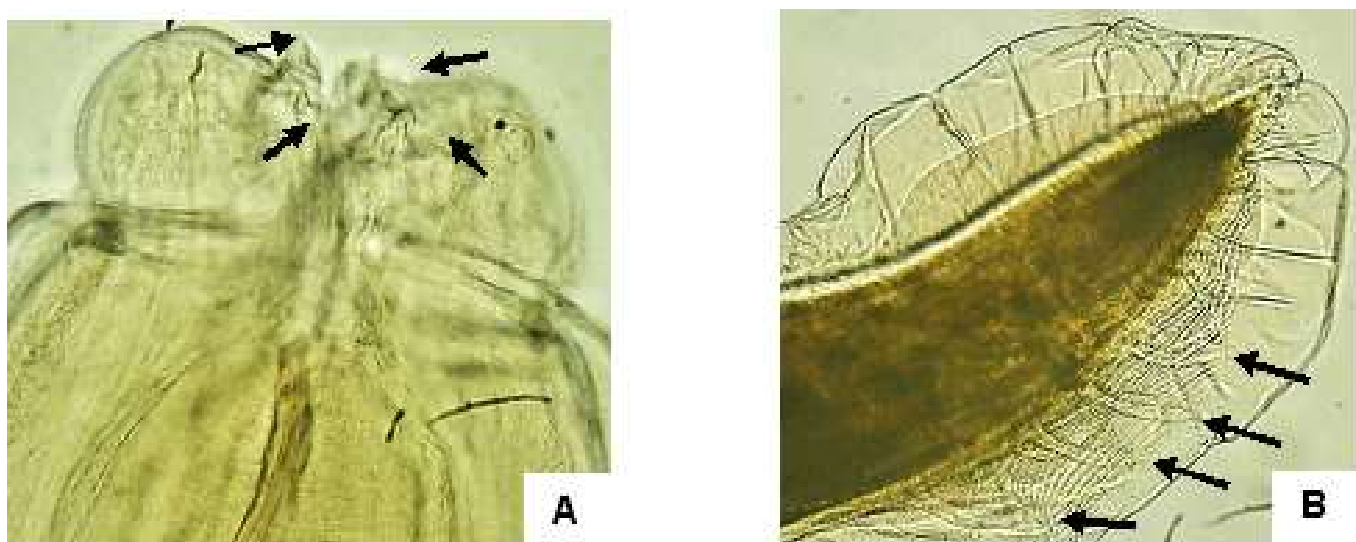

Figura 7. Mastophorus muris. A) Región anterior de Mastophorus muris. Vista lateral de la cápsula bucal, observándose varios dientes de diferentes tamaños (flechas) (100X). B) Vista dorsal de la región posterior del macho. Nótese las papilas caudales (flechas) (100X) 

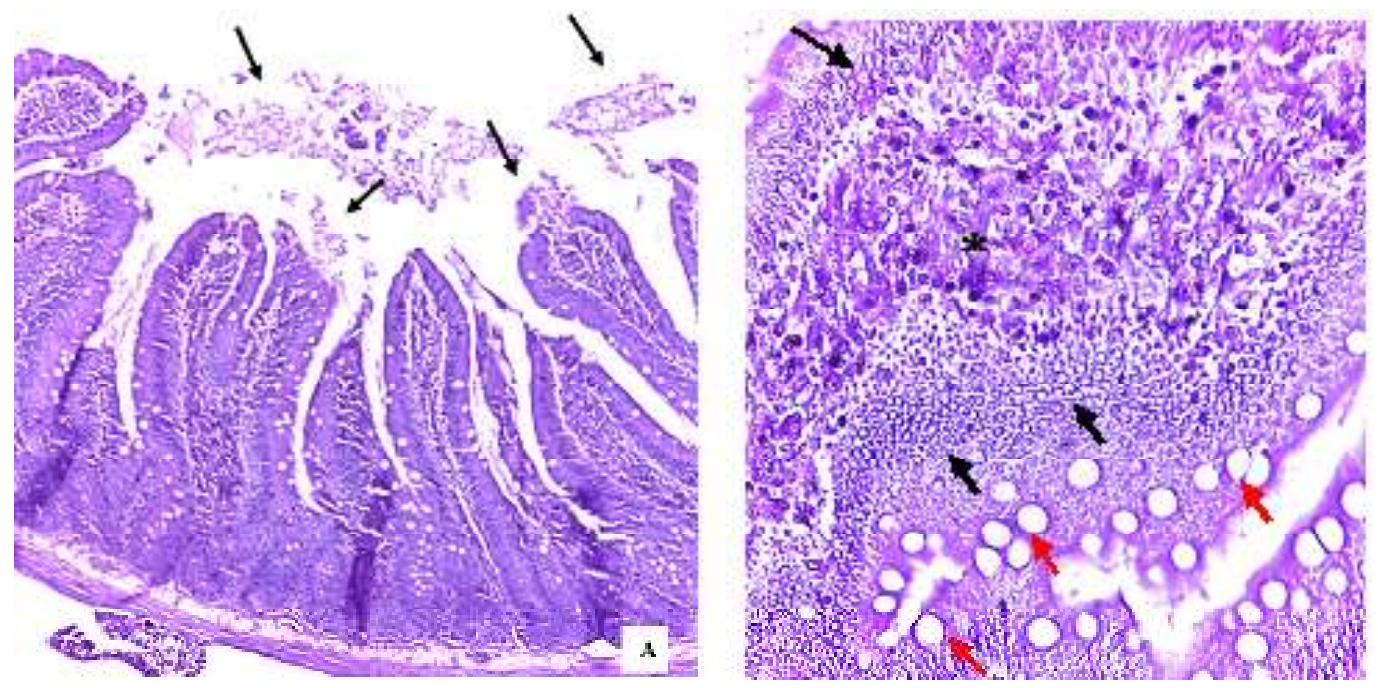

Figura 8. Lesiones microscópicas causadas por Hymenolepis diminuta. A. Disminución del tamaño de las vellosidades intestinales con desprendimiento de las células epiteliales de la mucosa (flechas negras) (100X). B. Aumento de las células caliciformes (flechas rojas) y enterocitos (flechas negras); en la lámina propia escasas células inflamatorias: eosinófilos y linfocitos (asterisco) (400X)

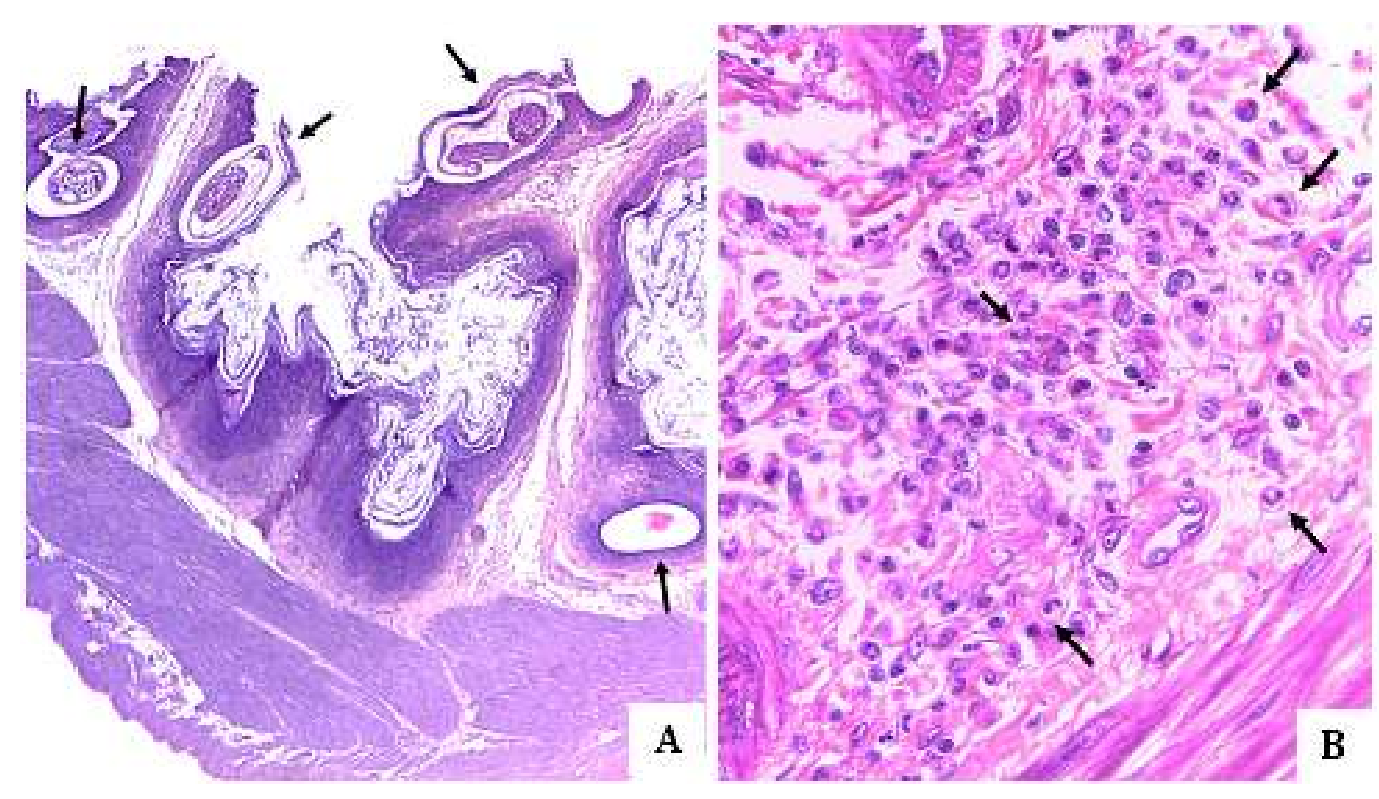

Figure 9. Lesiones histopatológicas causadas por Gongylonema neoplasticum. A. Se observa cuatro estructuras parasitarias, seccionadas transversalmente, embebidas en el epitelio escamoso estratificado queratinizado (flechas); y aumento con disgregamiento de las capas del estrato escamoso y córneo (hiperqueratosis) (40X). B. Infiltrado de un gran número de eosinófilos en la submucosa (flechas) (400X) 
El estudio reporta por primera vez en Perú, la presencia de Mastophorus muris (Figura 7) en $R$. rattus $(3.3 \%)$ y $R$. norvegicus $(6.3 \%)$ de granjas $(7.7 \%)$ y zoológicos (4.4\%), representando un $4.5 \%$ del parasitismo total, proponiéndose ambas especies como nuevos hospederos en Lima, Perú.

Dentro de los factores que podrían haber influido en la baja parasitosis en los roedores de los mercados en comparación con los roedores de granjas porcinas y zoológico, así como con estudios previos en mercados y otros ecosistemas de Lima, sería la presencia o ausencia de hospedadores intermediarios infectados en los mercados muestreados, el sistema inmunológico de las ratas y su baja densidad poblacional, considerando que un gran número de individuos genera un mayor hacinamiento y mayores probabilidades de infección (Kataranovski et al., 2011).

Así mismo, el menor número de animales capturados en mercados podría estar influenciado por las mejoras continuas que vienen realizando los municipios en los sistemas de alcantarillado, programas constantes de desratización (dos veces al año en promedio) y fumigación contra insectos. Adicionalmente, se observó la presencia de gatos en relación inversa a la presencia de ratas en los mercados. Por otro lado, las ratas de granjas y zoológico fueron capturadas con mucha mayor facilidad, posiblemente debido a que en estos lugares no se suele emplear el uso masivo y periódico de trampas y cebos para el control de estos roedores.

El escaso número de $R$. norvegicus en relación con $R$. rattus en los mercados del presente estudio coincide con el estudio de Romero et al. (2012), quienes capturaron 58 $R$. rattus y $3 R$. norvegicus en varios mercados de Lima. Estos valores pueden estar relacionados con el tipo de hábitat de estas especies, pues $R$. rattus se asocia típicamente con la presencia de basura, bodegas y alimentos de restaurante, ampliamente disponi- bles en los mercados (Mohd et al., 2012). Además, al ser buenas trepadoras tienden a vivir en lugares más elevados, facilitándose su dispersión a través de los cables y falsos techos de los mercados (Jarbas et al., 2002). En el caso de $R$. novergicus o rata de alcantarilla, su escaso número significaría que los problemas derivados de deficiencias en los desagües serían cada vez menores en estos lugares. No obstante, estos resultados son diferentes en granjas porcinas y en el zoológico, donde se logró capturar cantidades similares de $R$. rattus y $R$. norvegicus.

Las lesiones histopatológicas generadas en los tejidos afectados por los helmintos de potencial zoonótico (Hymenolepis diminuta [Figura 8], Moniliformis moniliformis y Raillietina demerariensis) son congruentes con los hallados por Movsesyan et al. (2008), Goswami et al. (2011) y Mahmoud et al. (2011), quienes reportaron degeneración y descamación celular, atrofia de vellosidades intestinales, inflamación de la lámina propia, hiperplasia de enterocitos y células caliciformes, infiltrado linfopalmocítico y eosinolfílicos e hiperplasia linfoide.

Reportes sobre los daños histopatológicos generados en el estómago de las ratas por espiruloideos son escasos y antiguos. Hall (1916) reportó hipertrofia de la mucosa con formación de papilomas y carcinomas con ocasional metástasis como consecuencia del parasitismo por Gongylonema neoplasticum. Sin embargo, en el presente estudio, se observó hiperqueratosis en las capas del estrato escamoso y córneo e infiltrado de un gran número de eosinófilos en la submucosa (Figura 9), sin presencia de lesiones neoplásicas aparentes; resultados coincidentes con los hallados por Bacigalupo (1929) en Argentina, donde reportó una prevalencia de $3.5 \%$.

La gran diversidad parasitaria hallada en las ratas de los ecosistemas en estudio demuestra la adaptabilidad y capacidad que poseen para soportar el parasitismo. Por otro lado, su capacidad de supervivencia, prolifera- 
ción y adaptabilidad a estos ecosistemas permiten que contaminen el medioambiente, agua y alimentos con parásitos de potencial zoonótico.

\section{Conclusiones}

- La prevalencia de helmintos gastrointestinales en ratas de tres medioambientes de la ciudad de Lima fue de $72.2 \%$, en tanto que la prevalencia de helmintos zoonóticos fue de $46.5 \%$.

- Las especies de potencial zoonótico hallados en granjas porcinas, zoológico y mercados de abastos fueron Hymenolepis diminuta $(53.8,8.8$ y 9.5\%, respectivamente), Moniliformis moniliformis $(9.9,38.5$ y $15.9 \%$, respectivamente), Gongylonema neoplasticum (4.4,9.9, $3.2 \%$, respectivamente) y Raillietina demerariensis ( $9.9 \%$ en granjas porcinas y $1.1 \%$ en el zoológico).

\section{Agradecimientos}

El estudio fue apoyado por la subvención del Fondo de Promoción de Trabajo de Tesis del Vicerrectorado de Investigación UNMSM. Los autores agradecen a la Mg. Rosa Martínez Rojas (Facultad de Biología UNMSM) por su apoyo con las técnicas parasitológicas de montaje y coloración; a la MV Katherine Robles Noriega (Facultad de Medicina Veterinaria - UNMSM) por su ayuda en el procesamiento, colección e identificación de los helmintos; y al Sr. Gerald Urbano Moreno Morales, miembro del Círculo de Investigación de Parasitología (CIPAR), por su apoyo en la captura, necropsia de roedores, colección de helmintos y análisis estadístico.

\section{Literatura Citada}

1. Anderson RC, Chabaud AG, Willmott S. 2009. Key to the nematode parasites of vertebrates. UK: CABI International. $463 \mathrm{p}$.
2. Ayulo V, Dammert O. 1947. Survey del parasitismo intestinal de las ratas grises (Mus novergicus) en la ciudad de Lima. Rev Perú Med Exp Salud Pública 6: 76-93.

3. Bacigalupo J. 1929. Contribución al estudio del Gongylonema neoplasticum (Fibiger-Ditlevsen) en la Argentina. Rev Ch Hist Nat 23: 414-417.

4. Baker D. 2007. Flynn's parasites of laboratory animals. $2^{a}$ ed. Iowa: Blackwell Publishing. $830 \mathrm{p}$.

5. Behmer OA, Tolosa EMC, Neto AGF. 1976. Manual de técnicas para histología normal e patológica. São Paulo: São Paulo Livraria Ed. 241 p.

6. Bowman DD. 2009. Georgis: parasitología para veterinarios. $9^{a}$ ed. Barcelona: Elsevier. 453 p.

7. Botero D, Restrepo M. 2006. Parasitosis humanas. $4^{\mathrm{a}}$ ed. Bogotá: Corporación para Investigaciones Biológicas. $506 \mathrm{p}$.

8. Daniel W. 1996. Bioestadística base para el análisis de las ciencias de la salud. $3^{\mathrm{a}}$ ed. México: Limusa. $875 \mathrm{p}$.

9. De Sotomayor R, Serrano-Martínez E, Tantaleán M, Quispe M, Casas $G$ 2015. Identificación de parásitos gastrointestinales en ratas de Lima Metropolitana. Rev Inv Vet Perú 26: 273281. doi: 10.15381/rivep.v26i2.11003

10. EL-Sayad MH, Lotfy WM. 2005. Effect of storage on the infectivity of Vampirolepis nana var. nana eggs to Swiss Albino mice. Iranian J Publ Health 34(4): 29-34.

11. Gómez-Villafañe I, Robles MR, Busch M. 2008. Helminth communities and host-parasite relationships in argentine Brown rat (Rattus norvegicus). Helminthologia 45: 126-129. doi: 10.2478/ s11687-008-0024-1

12. Goswami R, Mohan S, Kataria M, Somvanshi R. 2011. Clinicopathological studies on spontaneous Hymenolepis diminuta infection in wild and laboratory rats. Braz J Vet Pathol 4: 103-111.

13. Hall MC. 1916. Nematode parasites of mammals of the Orders Rodentia, Lagomorpha, and Hyracoidea. Proc US National Museum 50: 1-247. 
14. Haruki K, Furuya H, Saito S, Kamiya $S$, Kagei N. 2005. Gongylonema infection in man: a first case of gongylonemosis in Japan. Helminthologia 42: 63-66.

15. Iannacone J, Alvariño L. 2002. Helmintofauna de Rattus rattus (Linnaeus, 1758) y Rattus norvegicus (Berkenhout, 1769) (rodentia: muridae) en el distrito de San Juan de Lurigancho, Lima - Perú. Rev Peru Med Exp Salud Pública 19: 136-141.

16. Ibañez NH, Jara CC, Guerra AM. 2005. Helmintos parásitos de mamíferos silvestres de Condorcanqui, Amazonas-Perú. Rebiol 25: 43-48.

17. Jarbas B, Urbiratan P, Sadi C. 2002. Manual de control de roedores. Ministério da Saúde. Brazilia: Fundação Nacional de Saúde. 66 p.

18. Khalil LF, Jones A, Bray RA. 1994. Key to the cestode parasites of vertebrates. UK: CABI International. $768 \mathrm{p}$.

19. Kataranovski D, Kataranovski M, Sava IR, Soldatovice B, Matice R. 1994. Morphometric and biochemical parameters as the age indicators in the Norway rat (Rattus norvegicus Berk, 1769). Acta Vet 44: 371-378. doi: 10.1051/parasite/2011182189

20. Kataranovski M, Mirkov I, Belij S, Popov A, Petrovice Z, Gaèice Z, Kataranovski D. 2011. Intestinal helminths infection of rats (Ratus norvegicus) in the Belgrade area (Serbia): the effect of sex, age and habitat. Parasite 18: 189-196.

21. Macnish MG, Morgan UM, Behnke JM, Thompson RCA. 2002. Failure to infect laboratory rodent hosts with human isolates of Rodentolepis (=Hymenolepis) nana. J Helminthol 76: 37-43.

22. Mahmoud MS, El Namaky AH, Kandil OM, Allam NA, Hasan AA, Ashry HM. 2011. Advanced approach in differentiation study in Hymenolepis nana and $H$. Diminuta by scanning electron microscopy. Acta Parasitol Globalis 2(2): 34-39.

23. Martínez, R, Tantaleán M. 1998. Primer registro en el Perú de Raillietina (R.) demeraniensis (Cestoda: Taenioidea) en Rattus rattus norvegicus. Rev Per Biol 5: 65-68.

24. Milazzo C, Cagnin M, Di Bella C, Geraci F, Ribas $A$. 2010. Helminth fauna of commensal rodents, Mus musculus (Linnaeus, 1758) and Rattus rattus (Linnaeus, 1758) (Rodentia, Muridae) in Sicily (Italy). Rev Ibero-Latinoam. Parasitol 69: 194-198.

25. Mills J, Childs J, Ksiazek T, Peters CJ, Velleca WM. 1998. Métodos para trampeo y muestreo de pequeños mamíferos para estudio virológico. Organización Panamericana de la Salud, Washington, USA. 64 p. [Internet]. Disponible en: http://stacks.cdc.gov/view/cdc/11583

26. Mohd S, Behnke J, Lewis J. 2012. Helminth communities from two urban rat populations in Kuala Lumpur, Malaysia. Parasit Vectors 5: 47. doi: 10.1186/1756-3305-5-47

27. Movsesyan SO, Jivanyan KA, Chubaryan FA, Malczewski A, Terenina NB, Petrossyan $R$, TerOrganyan KS. 2008. Experimental hymenolepiasis of rats: preliminary data on histopathological changes of visceral organs. Acta Parasitol 53: 193-196. doi: 10.2478/s11686-008-0023-x

28. Paramasvaran S, Sani RA, Hassan L, Hanjeet K, Krishnasamy M, John J, Santhana R, et al. 2009. Endo-parasite fauna of rodents caught in five wet markets in Kuala Lumpur and its potential zoonotic implications. Trop Biomed 26: 67-72.

29. Pakdel N, Naem S, Rezaei F, Chalehchaleh A. 2013. A survey on helminthic infection in mice (Mus musculus) and rats (Rattus norvegicus and Rattus rattus) in Kermanshah, Iran. Vet Res Forum 4: 105-109.

30. Parshad VR. 1999. Rodent control in India. Integrative Pest Manag Rev 4: 97-126. 
31. Rodríguez NF, Tejedor-Junco MT, Hernández-Trujillo Y, González M, Gutiérrez C. 2010. The role of wild rodents in the transmission of Trypanosoma evansi infection in an endemic area of the Canary Islands (Spain). Vet Parasitol 174: 323-327. doi: 10.1016/ j.vetpar.2010.09.001

32. Romero H, Tantaleán M, Martínez R, Sáez G. 2012. Helmintofauna en roedores (Rodentia: Muridae) en mercados del distrito del Rímac. En: Libro de Resúmenes VIII Congreso Peruano de Parasitología. Trujillo.

33. Stojcevic D, Mihaljevic Z, Marinculic A. 2004. Parasitological survey of rats in rural regions of Croatia. Vet Med Czech 49: 70-74.

34. Tantaleán VM. 1994. Nuevos helmintos de importancia médica en el Perú. Rev Per Med Trop UNMSM 8: 87-91.
35. Tantaleán VM, Sánchez L, Gómez L, Huiza A. 2005. Acantocéfalos del Perú. Rev Peru Biol 12: 83-92. doi: 10.15381/ rpb.v12i1.2361

36. Tung KCH, Hsiao F, Yang CH, Chou CH, Lee W, Wang K, Lai CH. 2008. Surveillance of endoparasitic infections and the first report of Physaloptera sp and Sarcocystis spp in farm rodents and shrews in central Taiwan. J Vet Med Sci 71:43-47.

37. Tung KCH, Hsiao F, Wang K, Yang CH, Lai CH. 2013. Study of the endoparasitic fauna of commensal rats and shrews caught in traditional wet markets in Taichumg City, Taiwan. J Microbiol Immunol Infect 46: 85-88. doi: 10.1016/j.jmii.2012.01.012 